\title{
Fibroblast Growth Factor 23: A Biomarker of Kidney Function Decline
}

\author{
David A. Drew ${ }^{a}$ Ronit Katz ${ }^{b}$ Stephen Kritchevsky ${ }^{c}$ Joachim H. Ix ${ }^{d}$ \\ Michael G. Shlipak ${ }^{e}$ Anne B. Newman ${ }^{f}$ Andy Hoofnagle ${ }^{b} \quad$ Linda Fried $^{g}$ \\ Mark J. Sarnak ${ }^{a}$ Orlando M. Gutierrez ${ }^{\text {h }}$ \\ ${ }^{a}$ Tufts Medical Center, Boston, MA, USA; ${ }^{b}$ University of Washington, Seattle, WA, USA; ${ }^{C}$ Wake Forest University, \\ Winston-Salem, NC, USA; ${ }^{d}$ University of California San Diego School of Medicine, San Diego, CA, USA; e University of \\ California San Francisco School of Medicine, San Francisco, CA, USA; ${ }^{f}$ University of Pittsburgh School of Medicine, \\ Pittsburgh, PA, USA; ' VA Pittsburgh Healthcare System, Pittsburgh, PA, USA; h University of Alabama School of \\ Medicine, Birmingham, AL, USA
}

\section{Keywords}

Fibroblast growth factor $23 \cdot$ Incident chronic kidney disease $\cdot$ Biomarker $\cdot$ Kidney function decline

\begin{abstract}
Background: Fibroblast growth factor 23 (FGF-23) is a hormone that regulates phosphorus levels and vitamin $D$ metabolism. Previous studies have shown FGF-23 to be a risk factor for incident end-stage renal disease; however, there are less data on the association of FGF-23 with earlier kidneyrelated outcomes. Methods: Serum FGF-23 was assayed using an intact ELISA assay in 2,496 participants of the Healthy Aging and Body Composition Study, a cohort of well-functioning older adults. Kidney function was estimated by assaying cystatin $C$ at baseline and years 3 and 10. The associations between FGF-23 and decline in kidney function (defined by estimated glomerular filtration rate (eGFR) decline $\geq 30 \%$ or $\geq 3 \mathrm{~mL} / \mathrm{min} /$ year) and incident chronic kidney disease (CKD; incident eGFR $<60 \mathrm{~mL} / \mathrm{min} / 1.73 \mathrm{~m}^{2}$ and $\geq 1 \mathrm{~mL} / \mathrm{min} /$ year decline) were evaluated. Models were adjusted for demographics, baseline eGFR, urine albumin/creatinine ratio, comorbidity, and serum calcium, phosphorus, $25(\mathrm{OH})$ vitamin $\mathrm{D}$ and
\end{abstract}

() 2018 S. Karger AG, Basel parathyroid hormone. Results: The mean (SD) age was 75 (3) years, with $52 \%$ female and $38 \%$ black. There were 405 persons with $30 \%$ decline, 702 with $>3 \mathrm{~mL} / \mathrm{min} /$ year decline, and 536 with incident CKD. In fully adjusted continuous models, doubling of FGF-23 concentrations was not associated with kidney function decline $(\mathrm{OR}[95 \% \mathrm{Cl}]=0.98[0.82-1.19]$ for $\geq 30 \%$ decline and OR 1.17 [95\% Cl 1.00-1.37] for $\geq 3 \mathrm{~mL} / \mathrm{min} /$ year decline), or incident CKD (incident rate ratio [IRR] 1.05 [95\% Cl 0.91-1.22]). In adjusted quartile analysis, the highest quartile of FGF-23 was significantly associated with incident CKD (IRR 1.27 [95\% Cl 1.02-1.58] for highest vs. lowest quartile). Conclusion: Higher FGF-23 concentrations were not consistently associated with decline in kidney function or incident CKD in community-dwelling older adults.

(c) 2018 S. Karger AG, Basel

\section{Introduction}

Fibroblast growth factor 23 (FGF-23), a hormone secreted by bone, serves as a regulator of phosphorus balance [1] and vitamin D metabolism [2]. A physiologic rise in FGF-23 is increasingly recognized as one of the earliest

\section{KARGER}

E-Mail karger@karger.com www.karger.com/ajn
David A. Drew, MD, MS

Tufts Medical Center

Boston, MA 02111 (USA)

E-Mail ddrew@tuftsmedicalcenter.org 
metabolic abnormalities that occurs in chronic kidney disease (CKD) [3], with increases seen even with relatively preserved kidney function [4]. Although increases in FGF-23 are felt to be an appropriate physiologic response to maintain normal phosphorus balance [5], elevated FGF-23 concentrations result in the following outcomes: mortality [6], cardiovascular events [7], and end-stage renal disease (ESRD) [6, 8-10].

There is increasing interest in discovering novel biomarkers of kidney function decline, particularly in discovering those that may provide valuable prognostic information in the earlier stages of CKD or in the general population. Given the association between high FGF-23 and ESRD, FGF-23 has been hypothesized to be one such biomarker. However, there are relatively fewer data on the association between FGF-23 with more proximate kidney outcomes such as incident CKD [10-12]. Given the association seen with ESRD, we hypothesized that high FGF-23 concentrations would be associated with decline in eGFR as well as with incident CKD, independent of baseline kidney function. Therefore, within the Health Aging and Body Composition Study (Health $\mathrm{ABC}$ ), a large diverse cohort of elderly well-functioning adults with repeated measures of cystatin $\mathrm{C}$, we assayed intact FGF-23 in order to determine the association of FGF-23 with subsequent decline in kidney function and the development of incident CKD.

\section{Materials and Methods}

\section{Study Population}

Health ABC is a prospective cohort initiated in 1997 with a goal of assessing how health conditions impact age-related physiologic and functional status. The study population consists of 3,075 participants aged 70-79 years at baseline with an equal number of men and women and $38 \%$ black. All persons included were determined to be free of disability in activities of daily living and free of functional limitation at baseline. All participants who had measures of FGF-23 at year 2 (stored samples were not available for testing from the baseline visit) and kidney function at baseline, and at least one repeat measure of kidney function at either year 3 or year 10 $(n=2,496)$ were included in the current study. All participants provided written consent for the study. The study was approved by institutional review boards at each participating institution and meets the requirements of the Declaration of Helsinki.

Exposure

FGF-23 was measured using a commercial ELISA that detects the full-length intact peptide (Kainos Laboratories, Japan). Serum samples from the 2 nd year visit were stored at $-70{ }^{\circ} \mathrm{C}$ and were not thawed until the time of analysis. This particular FGF-23 assay has a limit of detection of $3 \mathrm{pg} / \mathrm{mL}$. Samples were assayed in batches over 2 months and the between-batch coefficient of variation was $10.7 \%$.

\section{Covariates}

All covariates were obtained at study enrollment, with the exception of measures of mineral metabolism, which were assayed in samples obtained from the 2 nd year visit. Cardiovascular disease (CVD) status was defined as a prior history of coronary artery disease, stroke, or heart failure. Diabetes was defined as use of hypoglycemic agents, self-reported history, fasting plasma glucose level $\geq 126 \mathrm{mg} / \mathrm{dL}$, or 2 -h oral glucose tolerance test result $\geq 200 \mathrm{mg} / \mathrm{dL}$. Systolic and diastolic blood pressures were obtained by trained and certified clinical staff from the right arm using a conventional mercury sphygmomanometer with the participant in a seated position. Hypertension was defined as systolic blood pressure $\geq 140 \mathrm{~mm} \mathrm{Hg}$, diastolic blood pressure $\geq 90 \mathrm{~mm} \mathrm{Hg}$, or current use of antihypertensive medications and a self-reported physician's diagnosis of hypertension. Urine albumin to creatinine ratio (UACR) was determined at baseline. Urine albumin was measured using a particle-enhanced turbidimetric inhibition immunoassay allowing for direct albumin quantification (Siemens), while urine creatinine was measured by a modified Jaffé method on a clinical chemistry analyzer (Siemens). Measures of mineral metabolism including calcium, phosphorus, $25(\mathrm{OH})$ vitamin $\mathrm{D}$, and parathyroid hormone $(\mathrm{PTH})$ were measured at year 2, concurrent with FGF-23 measurement, from frozen stored samples. Intact PTH was measured in EDTA plasma using a 2-site immunoradiometric assay kit (N-tact PTHSP; DiaSorin). Serum calcium and phosphate levels were measured using direct quantitative colorimetric determination (Stanbio Laboratory, Boerne, TX, USA). Serum 25(OH) vitamin D was measured using a 2-step radioimmunoassay (25-Hydroxyvitamin D 125I RIA Kit, DiaSorin, Stillwater, MN, USA) in a laboratory meeting the vitamin D External Quality Assessment Scheme quality criteria.

\section{Outcomes}

Cystatin C was measured at baseline, as well as years 3 and 10 from stored frozen serum samples at the Health ABC core laboratory (University of Vermont, Burlington, VT, USA) using a BNII nephelometer (Dade Behring Inc., Deerfield, IL, USA) using a particle-enhanced immunonepholometric assay (N Latex Cystatin C) [13]. As in prior Health ABC studies [14,15], serum cystatin $C$ was used as the primary measure of kidney function rather than serum creatinine for 2 reasons. First, cystatin $\mathrm{C}$ measures were calibrated across all samples, whereas there was a shift in the creatinine assay from non-IDMS traceable (baseline) to IDMS traceable during the study (years 3 and 10). Second, cystatin $C$ is less influenced by age, sex, and race [16] and in particular muscle mass and has been shown to be more strongly associated with adverse outcomes in the elderly population $[17,18]$. Among 61 healthy individuals with 3 cystatin $\mathrm{C}$ measurements over a 6 -month period, the intra-individual coefficient of variation was $7.7 \%$, reflecting long-term stability of the measurement. Estimated glomerular filtration rate (eGFR) was calculated using a validated cystatin C-based estimating equation $[13,19]$. Using eGFR, 3 kidney outcomes were defined as follows.

\section{Change in Kidney Function}

(a) Relative decline in kidney function was defined as an eGFR decline of $30 \%$ or greater. When 2 follow-up measures of eGFR were available, the last available value (year 10) was utilized. Recent publications and a recent workshop between the United States Food and Drug Administration and National Kidney Foundation 
have argued that a $30 \%$ decline in GFR is an acceptable clinical endpoint for clinical trials, as it strongly and consistently predicts the development of ESRD [20, 21].

(b) Absolute decline in kidney function was defined as a decline in eGFR of $>3 \mathrm{~mL} / \mathrm{min} /$ year [22]. The rate of decline per year was determined by creating a slope using either 2 (single follow-up eGFR) or 3 ( 2 follow-up eGFR) data points. This definition has been used in prior publications $[14,15]$ and has been shown to be associated with mortality in older adults [22].

(c) Incident $\mathrm{CKD}$ was defined as an eGFR of $<60 \mathrm{~mL} /$ $\mathrm{min} / 1.73 \mathrm{~m}^{2}$ at any point during follow-up and an annual absolute decline $>1 \mathrm{~mL} / \mathrm{min} / 1.73 \mathrm{~m}^{2}$ per year, with the latter requirement in place to avoid inclusion of participants with minor changes in eGFR. Participants with an eGFR $<60 \mathrm{~mL} / \mathrm{min} / 1.73 \mathrm{~m}^{2}$ at baseline were excluded from this analysis.

\section{Statistical Analysis}

We examined baseline characteristics of participants across quartiles of FGF-23. These were summarized with means and standard deviations, or medians and interquartile ranges for highly skewed variables or proportions for categorical variables. For eGFR and UACR, we also included the proportion of participants in each quartile below or above clinically relevant cutoff points (eGFR $<60 \mathrm{~mL} / \mathrm{min} / 1.73 \mathrm{~m}^{2}$, UACR $>30 \mathrm{mg} / \mathrm{g}$ ). To examine the functional form of the association between FGF-23 and each outcome, we fitted unadjusted natural cubic splines with knots placed at the quartiles and extreme values excluded so as to avoid implausible extrapolation of shapes of the association.

\section{FGF-23 and Change in Kidney Function}

Multivariable logistic regression models were used to assess the relationship between FGF-23 and 30\% decline in eGFR as well as $>3 \mathrm{~mL} / \mathrm{min} /$ year decline in eGFR. FGF-23 was examined as both a continuous variable (log base 2 , so that interpretation would be "per doubling" of the exposure) and categorized by quartiles. Model 1 was unadjusted. Multivariable models were then sequentially constructed through a series of nested models using pre-specified variables as follows: model 2: adjusted for age, sex, race, study site, and baseline eGFR; model 3: additionally adjusted for diabetes, prevalent CVD, hypertension, and urine ACR; model 4: additionally adjusted for calcium, phosphorus, $25(\mathrm{OH})$ vitamin $\mathrm{D}$, and PTH. Based on a priori hypotheses that a synergistic relationship could potentially exist between FGF-23 and baseline eGFR (higher FGF-23 and lower eGFR), we evaluated if the association between FGF-23 and decline in GFR was modified by baseline eGFR by including interaction terms (with both FGF-23 and eGFR as continuous terms) in the final multivariable models. As black patients are at a higher risk for decline in kidney function [23], we also evaluated if associations differed by race by including race as an interaction term in the final models. To test if the presence of inflammation was a potential confounder, we performed a sensitivity analysis in which baseline serum C-reactive protein concentration was added to the final model. Finally, we performed a sensitivity analysis in which we determined the association between FGF-23 and $\geq 40 \%$ decline in eGFR.

\section{FGF-23 and Incident CKD}

Poisson (log-link) regression was used to model the incidence rate ratio (IRR) of CKD as a function of FGF-23 concentration with robust variance estimation and an offset for follow- up time. Exposure variables were again examined as both linear terms and by category (quartiles) to assess for non-linear relationships based on our spline analysis. Identical multivariable models were constructed as described above, including interaction terms.

Analyses were conducted using SPSS (IBM Corp., Released 2015; IBM SPSS Statistics for Windows, Version 23.0. Armonk, NY, USA) and Stata (StataCorp LP, 2013; Stata Statistical Software: Release 13. College Station, TX, USA). A 2 -sided $p$ value of $<0.05$ was considered statistically significant for all analyses including interaction terms.

\section{Results}

\section{Baseline Characteristics}

Among 3,075 persons, 300 participants did not have sample available for assaying FGF-23, while 279 were missing values for cystatin $\mathrm{C}$ at either baseline or both follow-up visits, leaving 2,496 with complete data available for analysis. Fifty-four percent of the remaining participants had 2 follow-up measures of eGFR (years 3 and 10 ), while $43 \%$ had a single follow-up eGFR measure from year 3 and $3 \%$ had a single follow-up eGFR measure from year 10. The median FGF-23 level was $46 \mathrm{pg} / \mathrm{mL}$ (37-60 [25th-75th percentile]). The average (SD) age was 75 (3) years, with $52 \%$ female and $38 \%$ black (Table 1 ). The mean baseline eGFR was $73(18) \mathrm{mL} / \mathrm{min} / 1.73 \mathrm{~m}^{2}$ and $23 \%$ of participants had a baseline eGFR of less than 60 $\mathrm{mL} / \mathrm{min} / 1.73 \mathrm{~m}^{2}$.

Across quartiles of FGF-23, those in the highest quartile were more likely to have comorbid conditions such as diabetes, hypertension, coronary artery disease, and heart failure. In addition, those in the highest quartile also had lower baseline eGFR, were more likely to have a spot urine ACR $>30 \mathrm{mg} / \mathrm{g}$, and had higher serum calcium, phosphorus, and PTH concentrations. There were no observed differences in age, sex, and black race by category of FGF-23.

\section{Splines}

In unadjusted spline analysis, we saw no clear relationship between FGF-23 and decline in kidney function (Fig. 1). Higher FGF-23 appeared to be linearly associated with a higher incidence of CKD, but only when values were greater than approximately $50 \mathrm{pg} / \mathrm{mL}$.

\section{FGF-23 and Change in Kidney Function}

The median annual rate of decline for all included participants was $1.45 \mathrm{~mL} / \mathrm{min} /$ year $(-3.38$ to $0.06 \mathrm{~mL} /$ $\mathrm{min} /$ year for 25 th and 75 th percentiles). Four hundred 
Table 1. Baseline demographics and clinical characteristics by quartiles of FGF-23

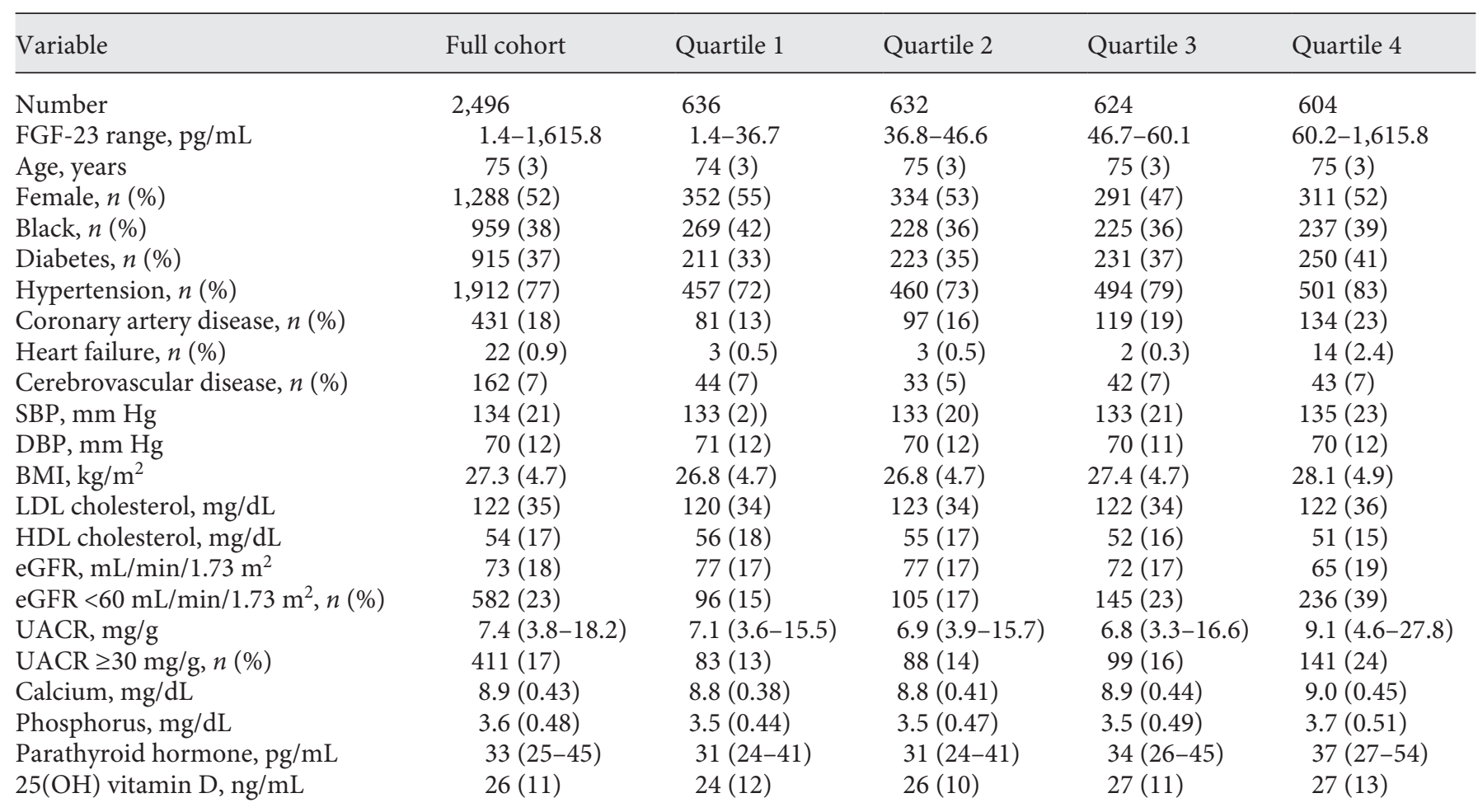

Presented as mean (SD), median (25th-75th percentile), or \% as appropriate.

FGF-23, fibroblast growth factor 23; SBP, systolic blood pressure; DBP, diastolic blood pressure; BMI, body mass index; LDL, low density lipoprotein; HDL, high density lipoprotein; UACR, urine albumin to creatinine ratio; eGFR, estimated glomerular filtration rate based on cystatin $\mathrm{C}$.

and 5 participants (16\%) experienced a relative 30\% decline in kidney function, while 702 (28\%) experienced an absolute decline in kidney function. Higher FGF-23 concentrations were not associated with a relative $(30 \%)$ decline in kidney function or with absolute decline (Table 2). This finding was unchanged throughout all multivariable models, including after adjustment for demographics, baseline kidney function, CKD and CVD risk factors, and measures of mineral metabolism (Table 2). Similarly, in sensitivity analyses using $\geq 40 \%$ decline in eGFR as the outcome variable, there was no significant association of FGF23 with eGFR decline in fully adjusted models (OR 1.10 [95\% CI 0.86-1.41]). In contrast, each twofold higher FGF-23 was associated with greater odds of absolute ( $>3 \mathrm{~mL} / \mathrm{min} /$ year) decline in kidney function in models adjusted for demographic variables, kidney function, and comorbid conditions, but not after further adjustment for measures of mineral metabolism (Table 2).

FGF-23: A Biomarker of Kidney Function Decline

\section{FGF-23 and Incident CKD}

Five hundred eighty 2 participants who had an eGFR $<60 \mathrm{~mL} / \mathrm{min} / 1.73 \mathrm{~m}^{2}$ at baseline were excluded from this analysis, leaving 1,914 participants available for analysis. Incident CKD was observed in 536 (25\%), with an incident rate of $4.8 \%$ per year (Table 3 ). Each twofold higher FGF-23 was associated with a $22 \%$ higher IRR for CKD, which lost statistical significance after adjustment for demographics and baseline eGFR. Across quartiles of FGF23 , those in the highest quartile demonstrated an IRR twice that of the lowest quartile (2.32, 95\% CI 1.45-3.74), which was attenuated but remained significant across subsequent models.

\section{Interactions and Sensitivity Analyses}

No significant interactions were noted between FGF23 and baseline eGFR with 30\% decline, absolute decline, and incident CKD (all $p$ values $>0.05$ ). Similarly, no interactions were found between FGF-23, race, and each kidney function outcome (all $p$ values $>0.05$ ). 


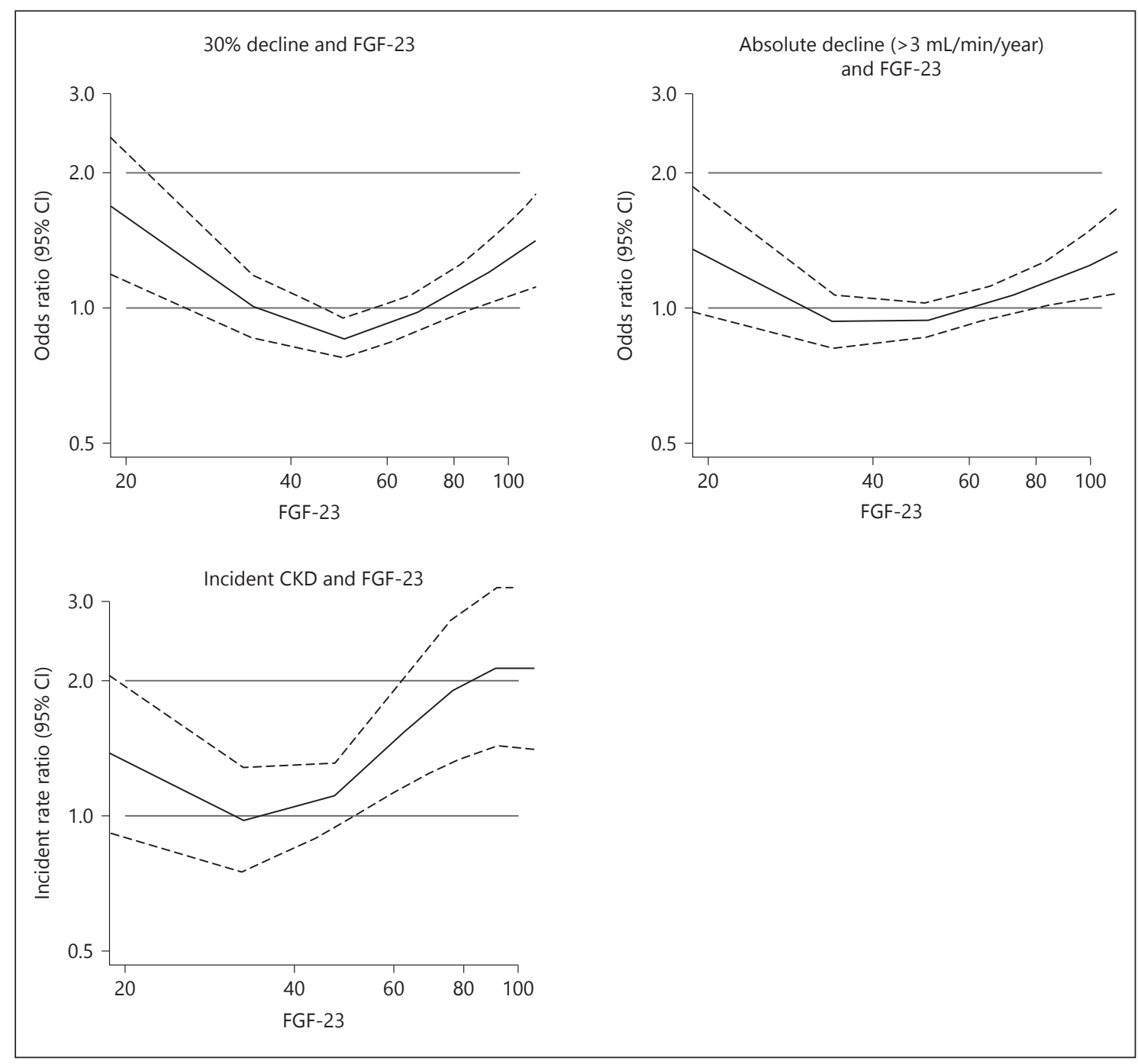

Fig. 1. Unadjusted association between FGF-23 and kidney function decline and incident CKD. FGF-23, fibroblast growth factor 23; CKD, chronic kidney disease.

Addition of C-reactive protein to the final adjusted model produced similar results for all 3 kidney outcomes.

\section{Discussion}

In a diverse cohort of older adults, the majority of whom did not have advanced kidney disease at baseline, we found an inconsistent association between higher FGF-23 and subsequent kidney-related outcomes. These relationships were largely attenuated when adjusting for comorbidity, kidney disease risk factors, and measures of mineral metabolism. Our results, particularly the at- tenuation of associations after adjustment for baseline eGFR, suggest that FGF-23 may be a marker of kidney health or function, rather than a mediator of kidney damage.

Multiple prior studies have shown a strong relationship between higher FGF-23 and subsequent risk of developing ESRD. Investigators of the Chronic Renal Insufficiency Study explored the relationship between FGF-23 and risk of ESRD in patients with moderate to advanced $\mathrm{CKD}$, finding that with each standard deviation higher FGF-23 was associated with a $30-70 \%$ higher adjusted hazard for ESRD, but only for those with baseline eGFR $>30 \mathrm{~mL} / \mathrm{min} / 1.73 \mathrm{~m}^{2}$ [9]. Additionally, post hoc analyses of the Homocysteine in Kidney and End-Stage Renal Dis- 
Table 2. Association of FGF-23 with kidney function decline in the Health ABC Study

\begin{tabular}{|c|c|c|c|c|c|c|}
\hline \multicolumn{7}{|l|}{$\geq 30 \%$ decline } \\
\hline$<36$ & 636 & 120 & 1.00 (ref.) & 1.00 (ref) & 1.00 (ref.) & 1.00 (ref.) \\
\hline$>60$ & 604 & 117 & $1.07(0.81-1.43)$ & $1.12(0.84-1.51)$ & $0.99(0.73-1.33)$ & $0.93(0.68-1.28)$ \\
\hline \multicolumn{7}{|l|}{$>3 \mathrm{~mL} / \mathrm{min} /$ year decline } \\
\hline Continuous (per doubling) & 2,496 & 702 & $1.11(0.96-1.28)$ & $1.28(1.10-1.49)$ & $1.21(1.04-1.41)$ & $1.17(1.00-1.37)$ \\
\hline \multicolumn{7}{|l|}{ FGF-23 quartiles } \\
\hline$<36$ & 636 & 183 & 1.00 (ref.) & 1.00 (ref.) & 1.00 (ref.) & 1.00 (ref.) \\
\hline $36-46$ & 632 & 165 & $0.87(0.68-1.12)$ & $0.87(0.68-1.13)$ & $0.86(0.66-1.12)$ & $0.85(0.65-1.10)$ \\
\hline \multicolumn{7}{|c|}{ Model 2 = adjusted for age, sex, race, study site, and baseline eGFR. } \\
\hline \multicolumn{7}{|c|}{ Model 3 = M2 + diabetes, cardiovascular disease, hypertension, and ACR. } \\
\hline \multicolumn{7}{|c|}{ Model 4 = M3 + calcium, phosphorus, 25(OH) vitamin D and PTH. } \\
\hline
\end{tabular}

Table 3. Association of FGF-23 with incident CKD (eGFR $<60 \mathrm{~mL} / \mathrm{min} / 1.73 \mathrm{~m}^{2}$ and $>1 \mathrm{~mL} / \mathrm{min} /$ year decline)*

\begin{tabular}{llllllll}
\hline & Numbers & $\begin{array}{l}\text { Incident } \\
\text { CKD }\end{array}$ & $\begin{array}{l}\text { Rate, } \\
\text { \%/years }\end{array}$ & $\begin{array}{l}\text { Model 1 } \\
\text { IRR (95\% CI) }\end{array}$ & $\begin{array}{l}\text { Model 2 } \\
\text { IRR (95\% CI) }\end{array}$ & $\begin{array}{l}\text { Model 3 } \\
\text { IRR (95\% CI) }\end{array}$ & $\begin{array}{l}\text { Model 4 } \\
\text { IRR (95\% CI) }\end{array}$ \\
\hline $\begin{array}{l}\text { FGF-23 (per doubling) } \\
\text { FGF-23 quartiles }\end{array}$ & 1,914 & 536 & 4.81 & $1.22(1.05-1.42)$ & $1.11(0.96-1.29)$ & $1.07(0.93-1.24)$ & $1.05(0.91-1.22)$ \\
$\quad<36$ & 540 & 140 & 4.37 & 1.00 (ref.) & $1.00($ ref.) & 1.00 (ref.) & $1.00($ ref.) \\
36-46 & 527 & 126 & 3.95 & $1.05(0.80-1.39)$ & $0.92(0.74-1.14)$ & $0.94(0.76-1.17)$ & $0.95(0.76-1.18)$ \\
$47-60$ & 479 & 133 & 4.80 & $1.40(0.99-1.99)$ & $0.99(0.80-1.22)$ & $1.01(0.82-1.24)$ & $0.99(0.80-1.24)$ \\
$>60$ & 368 & 137 & 6.94 & $2.32(1.45-3.74)$ & $1.33(1.07-1.65)$ & $1.29(1.04-1.60)$ & $1.27(1.02-1.58)$ \\
\hline
\end{tabular}

* Excludes those with eGFR $<60 \mathrm{~mL} / \mathrm{min} / 1.73 \mathrm{~m}^{2}$ at baseline.

Model $1=$ unadjusted analysis.

Model 2 = adjusted for age, sex, race, study site, and baseline eGFR.

Model 3 = M2 + diabetes, cardiovascular disease, hypertension, and ACR.

Model $4=\mathrm{M} 3$ + calcium, phosphorus, $25(\mathrm{OH})$ vitamin D and PTH.

IRR, incident rate ratio.

ease (HOST) study demonstrated that each SD increase in FGF-23 was associated with a $60 \%$ higher hazard for ESRD in fully adjusted models [8]. FGF-23 was also shown to be an independent risk factor for ESRD in the Atherosclerosis Risk in Communities cohort, in which the majority of patients did not have CKD at baseline [10].

The ARIC study also investigated the association between FGF-23 and incident CKD - which was defined based on a composite outcome of CKD-related Interna- tional Classification of Disease diagnosis codes for hospitalizations and deaths, USRDS-identified ESRD, or the development of an incident measured eGFR $<60$ $\mathrm{mL} / \mathrm{min} / 1.73 \mathrm{~m}^{2}$ and at least a $25 \%$ decline in eGFR from baseline - and found that higher FGF-23 was associated with incident $\mathrm{CKD}$, which was attenuated but remained statistically significant after adjusting for baseline kidney function and comorbidity (HR 1.03 [95\% CI 1.00-1.06]). The Action to Control Cardiovascular Risk in Diabetes Trial (ACCORD) also investigat- 
ed the relationship between FGF-23 and incident CKD in a case-cohort study of diabetic patients without CKD at baseline [12]. FGF-23 was associated with incident CKD in unadjusted analyses, but not when adjusting for clinical risk factors and baseline eGFR, with similar results seen for analyses examining the association of FGF-23 with eGFR slopes. In contrast to the ARIC and ACCORD findings, a smaller study of community dwelling, disabled, older women showed an independent association between FGF-23 and incident CKD (defined solely by clinical data based on estimated GFR), even in fully adjusted analyses [11]. None of these studies examined the association of FGF-23 with alternative measures of eGFR decline, such as a $30 \%$ decrease in eGFR from baseline or absolute decline in eGFR. Collectively, our data show that the associations between FGF-23 and less severe GFR decrements and incident CKD are weaker than those seen with ESRD and are therefore of unclear clinical significance.

There are multiple potential mechanisms that may explain the associations between FGF-23 and kidney-related outcomes. First, FGF-23 may simply be a sensitive biomarker of impaired kidney function or clearance as shown by its rise prior to other metabolic abnormalities seen in CKD $[3,24]$. Supporting this finding, a study by Shardlow et al. [25] showed that in a cohort of people with stage 3 CKD, FGF-23 was associated with all-cause mortality and $25 \%$ decline in eGFR in univariate analysis but not in multivariable models adjusting demographics, baseline eGFR, and UACR, as well as for $25(\mathrm{OH})$ vitamin $\mathrm{D}$ and PTH levels. Second, the association may be related to FGF-23's known physiologic mechanisms of action. Elevations in FGF-23 appear to occur in response to states of phosphorus excess such as CKD or high phosphorus intake, suggesting that FGF-23 may be a sensitive marker of overall phosphorus burden [1]. Since excess phosphorus is thought to be a potential promoter of dystrophic calcification, which can ultimately lead to kidney function decline, this may partly explain an association of FGF-23 with kidney function decline. FGF-23 has also been hypothesized to contribute toward kidney damage, either directly or via an indirect pathway related to the induction of cardiac hypertrophy and subsequent CVD $[26,27]$. FGF-23 levels also rise during episodes of acute kidney injury [28] (perhaps along with multi-organ dysfunction), which could provide an additional explanation as to why elevated FGF-23 concentrations may be more strongly associated with incident ESRD.

The use of an assay that detects only the intact FGF-23 peptide could also partially explain our results. This assay exclusively detects the intact form of FGF-23, as the 2 epitopes recognized by the ELISA are on either side of the proteolytic cleavage site [29]. A recent meta-analysis showed that studies utilizing the c-terminal assay, which detects intact FGF-23 as well as proteolytic fragments, demonstrate stronger associations with mortality compared to the Kainos assay [30]. The stronger c-terminal associations may in part be explained by confounding factors such as iron deficiency [31] and inflammation [32], which increase FGF-23 cleavage and lead to higher levels of FGF-23 fragments, without impacting intact FGF-23.

The strengths of this study include a large and diverse cohort, detailed ascertainment of risk factors and repeated measure of kidney function over a moderate follow-up time period. We also utilized an assay that measures only the biologically relevant intact FGF-23; use of the c-terminal assay may yield FGF-23 levels including fragments that are increased in states of inflammation [32]. There are also several limitations to the current study. We did not have simultaneous assessment of both the exposure (FGF-23 assayed at year 2) and baseline kidney function, though the measures were within 1 year of each other, and it is not apparent if within subject changes in FGF-23 over time [33] impact associations with clinical events including ESRD. Additionally, our cohort did not include any participants with advanced CKD at baseline, so we are unable to generalize our results to a higher risk population, which would almost certainly have higher baseline FGF-23 concentrations.

In summary, we found an association between FGF-23 and some but not all measures of kidney function decline. These results suggest that FGF-23 is not a strong independent biomarker for GFR decline or incident CKD. Further study is needed to determine potential mechanisms that may explain why the relationship is weaker for more proximate clinical outcomes.

\section{Acknowledgments}

This research was supported in part by the Intramural Research Program of the National Institutes of Health (NIH) and the National Institute on Aging (NIA) Contracts N01-AG-6-2101; N01-AG-6-2103; N01-AG-6-2106; NIA grant R01-AG028050, and NINR grant R01-NR012459. Dr. Drew was supported by NIDDK grant K23DK105327. Drs Shlipak, Sarnak, Ix, and Katz were supported by NIA grant 5R01AG027002-07. Dr. Orlando M. Gutierrez was supported by grant R01NS080850 and an American Heart Association Strategically Focused Research Network in Disparities grant. Dr. Andy Hoofnagle was supported by 
NORC grant P30 DK035816. The study sponsors had no role in study design; collection, analysis, and interpretation of the data; writing the report; and the decision to submit the report for publication; however, the NIA did approve the manuscript for submission.

\section{Disclosure Statement}

There are no conflicts of interest to declare. The results presented in this paper have not been published previously, in whole or in part, except in the abstract form.

\section{References}

1 Ferrari SL, Bonjour JP, Rizzoli R: Fibroblast growth factor-23 relationship to dietary phosphate and renal phosphate handling in healthy young men. J Clin Endocrinol Metab 2005;90:1519-1524.

2 Liu S, Tang W, Zhou J, Stubbs JR, Luo Q, Pi M, Quarles LD: Fibroblast growth factor 23 is a counter-regulatory phosphaturic hormone for vitamin D. J Am Soc Nephrol 2006; 17: 1305-1315.

- 3 Isakova T, Wahl P, Vargas GS, Gutiérrez OM, Scialla J, Xie H, Appleby D, Nessel L, Bellovich K, Chen J, Hamm L, Gadegbeku C, Horwitz E, Townsend RR, Anderson CA, Lash JP, Hsu CY, Leonard MB, Wolf M: Fibroblast growth factor 23 is elevated before parathyroid hormone and phosphate in chronic kidney disease. Kidney Int 2011;79:1370-1378.

4 Dhayat NA, Ackermann D, Pruijm M, Ponte B, Ehret G, Guessous I, Leichtle AB, Paccaud F, Mohaupt M, Fiedler GM, Devuyst O, Pechère-Bertschi A, Burnier M, Martin PY, Bochud M, Vogt B, Fuster DG: Fibroblast growth factor 23 and markers of mineral metabolism in individuals with preserved renal function. Kidney Int 2016;90:648-657.

5 Gutierrez O, Isakova T, Rhee E, Shah A, Holmes J, Collerone G, Jüppner H, Wolf M: Fibroblast growth factor-23 mitigates hyperphosphatemia but accentuates calcitriol deficiency in chronic kidney disease. J Am Soc Nephrol 2005;16:2205-2215.

- 6 Ix JH, Katz R, Kestenbaum BR, de Boer IH, Chonchol M, Mukamal KJ, Rifkin D, Siscovick DS, Sarnak MJ, Shlipak MG: Fibroblast growth factor-23 and death, heart failure, and cardiovascular events in communityliving individuals: CHS (Cardiovascular Health Study). J Am Coll Cardiol 2012;60: 200-207.

-7 Scialla JJ, Xie H, Rahman M, Anderson AH, Isakova T, Ojo A, Zhang X, Nessel L, Hamano T, Grunwald JE, Raj DS, Yang W, He J, Lash JP, Go AS, Kusek JW, Feldman H, Wolf M; Chronic Renal Insufficiency Cohort (CRIC) Study Investigators: Fibroblast growth factor-23 and cardiovascular events in CKD. J Am Soc Nephrol 2014;25:349-360.

8 Kendrick J, Cheung AK, Kaufman JS, Greene T, Roberts WL, Smits G, Chonchol M; HOST Investigators: FGF-23 associates with death, cardiovascular events, and initiation of chronic dialysis. J Am Soc Nephrol 2011;22: 1913-1922.

-9 Isakova T, Xie H, Yang W, Xie D, Anderson $\mathrm{AH}$, Scialla J, Wahl P, Gutierrez OM, Steiger- walt S, He J, Schwartz S, Lo J, Ojo A, Sondheimer J, Hsu CY, Lash J, Leonard M, Kusek JW, Feldman HI, Wolf M; Chronic Renal Insufficiency Cohort (CRIC) Study Group: Fibroblast growth factor 23 and risks of mortality and end-stage renal disease in patients with chronic kidney disease. JAMA 2011;305: 2432-2439.

10 Rebholz CM, Grams ME, Coresh J, Selvin E, Inker LA, Levey AS, Kimmel PL, Vasan RS, Eckfeldt JH, Feldman HI, Hsu CY, Lutsey PL; Chronic Kidney Disease Biomarkers Consortium: Serum fibroblast growth factor-23 is associated with incident kidney disease. J Am Soc Nephrol 2015;26:192-200.

-11 Semba RD, Fink JC, Sun K, Cappola AR, Dalal M, Crasto C, Ferrucci L, Fried LP: Serum fibroblast growth factor-23 and risk of incident chronic kidney disease in older communitydwelling women. Clin J Am Soc Nephrol 2012;7:85-91.

12 Isakova T, Craven TE, Lee J, Scialla JJ, Xie H, Wahl P, Marcovina SM, Byington RP, Wolf M: Fibroblast growth factor 23 and incident CKD in type 2 diabetes. Clin J Am Soc Nephrol 2015;10:29-38.

13 Stevens LA, Coresh J, Schmid CH, Feldman HI, Froissart M, Kusek J, Rossert J, Van Lente F, Bruce RD 3rd, Zhang YL, Greene T, Levey AS: Estimating GFR using serum cystatin C alone and in combination with serum creatinine: a pooled analysis of 3,418 individuals with CKD. Am J Kidney Dis 2008;51:395406.

14 Goldenstein L, Driver TH, Fried LF, Rifkin DE, Patel KV, Yenchek RH, Harris TB, Kritchevsky SB, Newman AB, Sarnak MJ, Shlipak MG, Ix JH; Health ABC Study Investigators: Serum bicarbonate concentrations and kidney disease progression in community-living elders: the Health, Aging, and Body Composition (Health ABC) Study. Am J Kidney Dis 2014;64:542-549.

15 Madero M, Peralta C, Katz R, Canada R, Fried L, Najjar S, Shlipak M, Simonsick E, Lakatta E, Patel K, Rifkin D, Hawkins M, Newman A, Sarnak M; Health ABC Study: Association of arterial rigidity with incident kidney disease and kidney function decline: the Health $\mathrm{ABC}$ study. Clin J Am Soc Nephrol 2013;8:424433.

16 Shastri S, Katz R, Rifkin DE, Fried LF, Odden MC, Peralta CA, Chonchol M, Siscovick D, Shlipak MG, Newman AB, Sarnak MJ: Kidney function and mortality in octogenarians: Cardiovascular Health Study
All Stars. J Am Geriatr Soc 2012;60:12011207.

17 Deo R, Fyr CL, Fried LF, Newman AB, Harris TB, Angleman S, Green C, Kritchevsky SB, Chertow GM, Cummings SR, Shlipak MG; Health ABC study: Kidney dysfunction and fatal cardiovascular disease - an association independent of atherosclerotic events: results from the Health, Aging, and Body Composition (Health ABC) study. Am Heart J 2008; 155:62-68.

18 Shastri S, Tighiouart H, Katz R, Rifkin DE, Fried LF, Shlipak MG, Newman AB, Sarnak MJ: Chronic kidney disease in octogenarians. Clin J Am Soc Nephrol 2011;6:14101417.

19 Inker LA, Schmid CH, Tighiouart H, Eckfeldt JH, Feldman HI, Greene T, Kusek JW, Manzi J, Van Lente F, Zhang YL, Coresh J, Levey AS; CKD-EPI Investigators: Estimating glomerular filtration rate from serum creatinine and cystatin C. N Engl J Med 2012;367:20-29.

20 Coresh J, Turin TC, Matsushita K, Sang Y, Ballew SH, Appel LJ, Arima H, Chadban SJ, Cirillo M, Djurdjev O, Green JA, Heine GH, Inker LA, Irie F, Ishani A, Ix JH, Kovesdy CP, Marks A, Ohkubo T, Shalev V, Shankar A, Wen CP, de Jong PE, Iseki K, Stengel B, Gansevoort RT, Levey AS: Decline in estimated glomerular filtration rate and subsequent risk of end-stage renal disease and mortality. JAMA 2014;311:2518-2531.

21 Levey AS, Inker LA, Matsushita K, Greene $\mathrm{T}$, Willis $\mathrm{K}$, Lewis E, de Zeeuw D, Cheung AK, Coresh J: GFR decline as an end point for clinical trials in CKD: a scientific workshop sponsored by the National Kidney Foundation and the US Food and Drug Administration. Am J Kidney Dis 2014;64:821835.

22 Rifkin DE, Shlipak MG, Katz R, Fried LF, Siscovick D, Chonchol M, Newman AB, Sarnak MJ: Rapid kidney function decline and mortality risk in older adults. Arch Intern Med 2008; 168:2212-2218.

23 Krop JS, Coresh J, Chambless LE, Shahar E, Watson RL, Szklo M, Brancati FL: A community-based study of explanatory factors for the excess risk for early renal function decline in blacks vs whites with diabetes: the Atherosclerosis Risk in Communities study. Arch Intern Med 1999;159:1777-1783.

24 van Ballegooijen AJ, Rhee EP, Elmariah S, de Boer IH, Kestenbaum B: Renal clearance of mineral metabolism biomarkers. J Am Soc Nephrol 2016;27:392-397. 
25 Shardlow A, McIntyre NJ, Fluck RJ, McIntyre CW, Taal MW: Associations of fibroblast growth factor 23, vitamin D and parathyroid hormone with 5-year outcomes in a prospective primary care cohort of people with chronic kidney disease stage 3. BMJ open 2017;7:e016528.

26 Faul C, Amaral AP, Oskouei B, Hu MC, Sloan A, Isakova T, Gutierrez OM, Aguillon-Prada R, Lincoln J, Hare JM, Mundel P, Morales A, Scialla J, Fischer M, Soliman EZ, Chen J, Go AS, Rosas SE, Nessel L, Townsend RR, Feldman HI, St John Sutton M, Ojo A, Gadegbeku C, Di Marco GS, Reuter S, Kentrup D, Tiemann K, Brand M, Hill JA, Moe OW, Kuro-o M, Kusek JW, Keane MG, Wolf M: FGF23 induces left ventricular hypertrophy. J Clin Invest 2011;121:4393-4408.

27 Gutierrez OM, Januzzi JL, Isakova T, Laliberte K, Smith K, Collerone G, Sarwar A, Hoff- mann U, Coglianese E, Christenson R, Wang TJ, deFilippi C, Wolf M: Fibroblast growth factor 23 and left ventricular hypertrophy in chronic kidney disease. Circulation 2009;119: 2545-2552.

28 Leaf DE, Wolf M, Waikar SS, Chase H, Christov M, Cremers S, Stern L: FGF-23 levels in patients with AKI and risk of adverse outcomes. Clin J Am Soc Nephrol 2012;7:12171223.

29 Yamazaki Y, Okazaki R, Shibata M, Hasegawa Y, Satoh K, Tajima T, Takeuchi Y, Fujita T, Nakahara K, Yamashita T, Fukumoto S: Increased circulatory level of biologically active full-length FGF-23 in patients with hypophosphatemic rickets/osteomalacia. J Clin Endocrinol Metab 2002;87:49574960.

30 Xiao Y, Luo X, Huang W, Zhang J, Peng C: Fibroblast growth factor 23 and risk of all- cause mortality and cardiovascular events: a meta-analysis of prospective cohort studies. Int J Cardiol 2014;174:824-828.

31 Wolf M, White KE: Coupling fibroblast growth factor 23 production and cleavage: iron deficiency, rickets, and kidney disease. Curr Opin Nephrol Hypertens 2014;23:411419.

32 David V, Martin A, Isakova T, Spaulding C, Qi L, Ramirez V, Zumbrennen-Bullough KB, Sun CC, Lin HY, Babitt JL, Wolf M: Inflammation and functional iron deficiency regulate fibroblast growth factor 23 production. Kidney Int 2016;89:135-146.

33 Bouma-de Krijger A, Bots ML, Vervloet MG, Blankestijn PJ, ter Wee PW, van Zuilen AD, Wetzels JF: Time-averaged level of fibroblast growth factor-23 and clinical events in chronic kidney disease. Nephrol Dial Transplant 2014;29:88-97. 